\title{
AUTOMATIC QUERY REWEIGHTING USING CO-OCCURRENCE GRAPHS
}

\author{
Billel Aklouche ${ }^{1,2,4}$, Ibrahim Bounhas ${ }^{1,4}$ and Yahya Slimani ${ }^{1,3,4}$ \\ ${ }^{1}$ LISI Laboratory of Computer Science for Industrial System, INSAT, Carthage University, Tunis, Tunisia \\ ${ }^{2}$ National School of Computer Science (ENSI), La Manouba University, Manouba, Tunisia \\ ${ }^{3}$ Higher Institute of Multimedia Arts of Manouba (ISAMM), La Manouba University, Manouba, Tunisia \\ ${ }^{4}$ JARIR: Joint group for Artificial Reasoning and Information Retrieval (www.jarir.tn)
}

\begin{abstract}
Providing a relevant response to the user has always been challenging. Query reformulation methods have been widely applied in an attempt to provide a better representation of the user's query and thus improve retrieval performance. In this paper, we present a new query reweighting method for document retrieval based on term co-occurrence graphs, which are built using a context window-based approach over the entire corpus. We propose an adapted version of the well-established Okapi BM25 model that allows identifying the most informative terms in the query and assigning them optimal weights. This measure stands out by its ability to evaluate the discriminative power of terms from co-occurrence graphs. Experimental results on two standard ad-hoc TREC collections show that our method improves both retrieval effectiveness and robustness and outperforms the state-of-the-art baselines with significant margins.
\end{abstract}

\section{KEYWORDS}

Query Reformulation, Query Reweighting, Co-occurrence Graph, BM25, Term's Discriminative Power, Ad-Hoc Information Retrieval

\section{INTRODUCTION}

The significant growth of information available nowadays requires increasingly powerful search systems, which allow to discern relevant information effectively among thousands, even hundreds of thousands of documents. The main objective in information retrieval (IR) is to provide the user relevant documents according to a specific need. A great deal of research has been conducted in an attempt to improve retrieval performance. One of the primary research areas in IR is query reformulation or query modification, which refers to techniques that reformulate the original query, in order to enhance retrieval results.

There are two major categories of query reformulation methods, namely: query expansion and query reweighting. The former group of methods attempts to improve retrieval effectiveness by adding new terms to the original query (Carpineto and Romano, 2012). On the other hand, the purpose of query reweighting methods is to modify the weights of terms in the original query without adding new ones, so that the most important terms in the query will be assigned higher weights (Karisani et al., 2016). However, a main challenge in query reformulation methods is the selection of good discriminative terms that allow retrieving relevant documents and do not hurt retrieval performance.

The BM25 probabilistic model (Robertson and Walker, 1994) is a robust and effective state-of-the-art IR model, which has been widely applied, especially in TREC (Text REtrieval Conference) experiments (https://trec.nist.gov). It allows measuring query-document similarity based on a set of complementary parameters, which includes the document length, the local distribution of terms within documents and their global distribution over the entire collection. Since its inception, numerous research studies have proposed modifications and improvements to the BM25 model in an attempt to enhance retrieval effectiveness (Rasolofo and Savoy, 2003; Song et al., 2008; Robertson and Zaragoza, 2009; Lv and Zhai, 2011a; He et al., 2011; Lv and Zhai, 2011b; Ariannezhad et al., 2017). Nevertheless, despite the enhancements that can be introduced to IR models, the key factor in controlling retrieval performance remains the set of query terms. 
Co-occurrence statistics allow to express and discover semantic relationships between terms (Carpineto and Romano, 2012). However, building a term co-occurrence graph and exploring its links is a challenging problem, especially for large graphs. A primary limitation is due to the manner in which the similarity between terms is measured (Peat and Willett, 1991). In this paper, we propose a new query reweighting method for document retrieval based on term co-occurrence graphs. We present an adapted version of the BM25 model that allows to measure relatedness between terms in co-occurrence graphs and to evaluate their discriminative power. It allows to identify the most informative query terms and to penalize trivial terms. The term co-occurrence graph is constructed using a context window-based approach over the entire corpus.

We evaluate the proposed method on two TREC collections, namely: the standard TREC Robust04 collection with 249 queries and the newest TREC Washington Post collection with 50 queries. Experimental results show that our proposal outperforms the state-of-the-art baselines by significant margins.

The remainder of the paper is organized as follows. We discuss some related work in Section 2. Section 3 outlines the proposed query reweighting method. In Section 4, we present the experimental design and discuss the results. The last section concludes the paper and provides insights for future work.

\section{RELATED WORK}

Several methods of query reformulation have been proposed in an attempt to obtain better results. In the literature, query reformulation methods can be categorized into two main classes: query expansion and query reweighting (Karisani et al., 2016). Here, we briefly review some studies on query reweighting. Indeed, there are two approaches for reformulating a query, namely the local approach and the global approach. The former relies on the use of a local analysis of top documents from the initial retrieval results, mostly using pseudo-relevance feedback (PRF) where the top $k$ ranked documents are considered relevant. Whereas in a global approach, there is no need for an initial retrieval round. The source used is the entire corpus.

One of the first research studies on query reweighting was proposed by Robertson and Jones (1976). The proposed approach is based on the relevance probability. The authors examined the use of statistical techniques to assign weights to the query terms by analyzing their distribution in documents. Indeed, the well-known term frequency-inverse document frequency (TF-IDF) weighting scheme was introduced on the basis of successive refinements of this work (Salton and Buckley, 1988). In Kim et al. (2001), authors proposed a hybrid method for expanding and reweighting queries. They considered the term co-occurrence similarity between the original query and the set of feedback documents. The new weights of the query terms are obtained using fuzzy inference. Authors in Chang and Chen (2006) examined the use of genetic algorithms for query reweighting based on the user's relevance feedback. Their experimental results on a small dataset showed improvements in terms of recall and precision. In Bendersky and Croft (2008), authors have demonstrated the effectiveness of a supervised method for discovering key concepts in verbose natural language queries and weighting them. They used query-dependent, corpus-dependent, and corpus-independent features to learn how to automatically extract the most important terms from long queries. The proposed method was shown to be more accurate than standard weighting methods such as the IDF measure. More recently, authors in Karisani et al. (2016) proposed a local feedback query reweighting method to detect and identify the most informative terms in queries and reweight them. They used the context in the initial set of top pseudo-relevant documents to extract meaningful information and assign optimal weights to the query terms based on their occurrence in that set of documents. The obtained results showed that the proposed method outperforms traditional baselines on the English and Persian languages.

Our proposal can be classified as a global query reformulation method. That is, we follow the second category of research on query reformulation and propose a new method for automatic query reweighting. We present the details of the proposed method in the following section.

\section{PROPOSED METHOD}

Figure 1 presents the architecture that implements our method. We first construct the term co-occurrence graph. Then, we proceed to query reweighting. Both steps are detailed in the following subsections. 


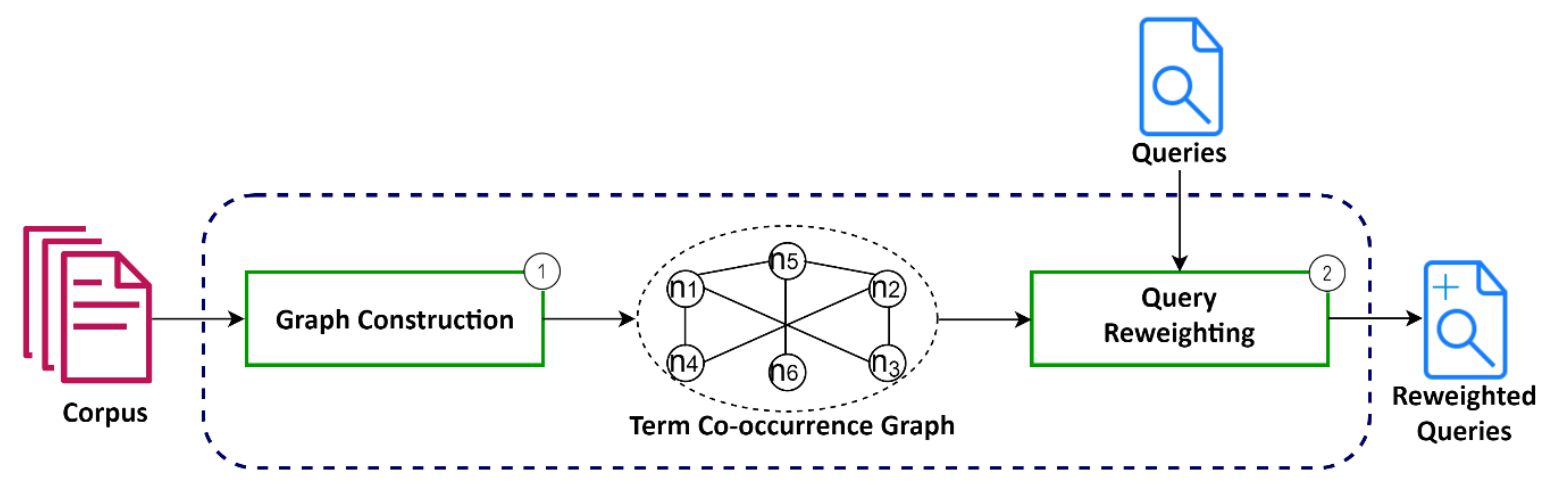

Figure 1. Overall architecture of the proposed method

\subsection{Graph Construction}

The term co-occurrence graph is constructed by applying a context window-based technique over the entire corpus. This technique, which allows capturing semantic relations between terms, has been widely used in several IR and Natural Language Processing (NLP) applications such as word embedding (Mikolov et al., 2013). Each window links the target term to its neighboring terms. For example, given the sentence "The $16^{\text {th }}$ edition of the International Conference on Applied Computing will take place in Italy" and taking "Conference" as the target term within a window-size of two terms, the context terms we get are "the", "International", "on", "Applied"\}. The co-occurrence of terms in the corpus is measured by applying a sliding-window over sentences. Thereafter, the co-occurrence graph is constructed using the obtained co-occurrence statistics of terms. In this graph, nodes represent the distinct terms in the corpus, i.e. the vocabulary, and the edges between nodes correspond to the frequency of their co-occurrences.

\subsection{Query Reweighting}

After the construction of the term co-occurrence graph, we present a measure inspired by Okapi BM25, which leverages the performance of this state-of-the-art model and allows to evaluate the discriminative power of terms from the constructed graph.

Given a document $D$ and a query $Q$ composed of $m$ terms $\left(t_{1}, \ldots, t_{m}\right)$, the BM25 score is calculated as follows (Robertson and Walker, 1994):

$$
B M 25(Q, D)=\sum_{t \in Q} I D F(t) \times \frac{\left(k_{1}+1\right) \times t f_{t D}}{k_{1} \times\left(1-b+b \times \frac{d l}{a v g d l}\right)+t f_{t D}}
$$

where:

- $I D F(t)$ represents the Inverse Term Frequency and it is computed as follows:

$$
I D F(t)=\log \frac{N-d f_{t}+0.5}{d f_{t}+0.5}
$$

- $\quad t f_{t D}$ is the term frequency of term $t$ in document $D$, i.e. the number of times $t$ occurs in $D$.

- $\quad d l$ denotes the document length, i.e. the number of terms in the document.

- $\quad a v g d l$ is the average document length in the collection.

- $\quad N$ is the number of documents in the collection.

- $\quad d f_{t}$ is the number of documents in which the term $t$ appears.

- $\quad k_{l}$ and $b$ are free tuning hyper-parameters. 
We use an adapted version of the BM25 model, which allows to measure the similarity of terms in co-occurrence graphs. The query terms are assigned weights according to their importance to the entire query and their relation to the rest of the vocabulary. We formalize the calculation of the weight of terms in co-occurrence graphs as follows. Let $C=\left\{n_{1}, \ldots, n_{m}\right\}$ be the projection of the query $Q=\left\{t_{1}, \ldots, t_{m}\right\}$ on the co-occurrence graph $G$ and $n_{c}$ be a node in $G$ corresponding to a query term. Using an adapted version of BM25, noted BM25 $\operatorname{cog}$ (BM25 for co-occurrence graphs), we calculate the weight of each query term as follows:

$$
B M 25_{\text {cog }}\left(C, n_{c}\right)=\sum_{n_{i} \in C} \operatorname{INF}\left(n_{i}\right) \times \frac{\left(k_{1}+1\right) \times e\left(n_{i}, n_{c}\right)}{k_{1} \times\left(1-b+b \times \frac{\text { sum_e } e\left(n_{c}\right)}{\text { avgsum_e }}\right)+e\left(n_{i}, n_{c}\right)}
$$

where:

- $\operatorname{INF}\left(n_{i}\right)$ denotes the Inverse Node Frequency of $n_{i}$. It reflects how much a term is distributed over the others and allows to penalize terms that co-occur with many other terms, i.e. they will get low values, independently of the initial query. This is analogous to Inverse Term Frequency (IDF) in the original BM25 formula and it is calculated as follows:

$$
\operatorname{INF}\left(n_{i}\right)=\log \frac{N-\text { co_degree }\left(n_{i}\right)+0.5}{\text { co_degree }\left(n_{i}\right)+0.5}
$$

- $e\left(n_{i}, n_{c}\right)$ is the weight of the edge between the nodes $n_{i}$ and $n_{c}$, that is, the number of co-occurrences of $n_{i}$ and $n_{c}$. This is analogous to the frequency of a term in a document. The main hypothesis behind this parameter is that terms that tend to co-occur frequently are likely to be similar.

- $\quad s u m \_e\left(n_{c}\right)$ is the sum of the weights of the edges linked to $n_{c}$. This is analogous to the document length parameter in query-document matching.

- $\quad$ avgsum_e is the average of the preceding parameter (i.e. sum_e $\left(n_{c}\right)$ ) for all the nodes in $G$. This is analogous to the average document length in the classical version of BM25.

- $\quad N$ is the number of nodes in $G$, which is analogous to the number of documents contained in the collection.

- co_degree $\left(n_{i}\right)$ is the number of nodes in $G$ co-occurring with $n_{i}$. In term-document graph, this is similar to the number of documents in which a given term appears.

- $\quad k_{1}$ and $b$ are the usual BM25 free hyper-parameters.

This measure allows to evaluate the discriminative power of terms. Thus, favor important terms in the query, which are both related to each other and have a relatively rare co-occurrence with the rest of the vocabulary. It allows penalizing trivial terms, which tend to co-occur with too many other terms. Besides, it has two free hyper-parameters that can be adjusted to improve results. Moreover, it has been successfully used for query expansion (Aklouche et al., 2019a, 2019b).

\section{EXPERIMENTS}

In this section, we first describe our datasets and experimental settings. Then we discuss the obtained results.

\subsection{Experimental Setup}

We used two ad-hoc TREC collections in our experiments, namely: the standard TREC Robust04 collection and the new TREC Washington Post collection ${ }^{1}$. The first collection is available in TREC disks 4 and $5^{2}$. It was used in the TREC 2004 Robust Track, which focused on poor performing queries. It consists of

\footnotetext{
${ }^{1}$ https://trec.nist.gov/data/wapost/

2 https://trec.nist.gov/data/cd45/
} 
newswire articles from different newspapers (Financial Times, Federal Register 94, Foreign Broadcast Information Service and LA Times) covering 1989 through 1996. The second collection was introduced in the TREC 2018 Common Core Track. It consists of news articles and blog posts collected from the Washington Post journal from 2012 to 2017. The statistics of these datasets are provided in Table 1.

Table 1. Test collections statistics

\begin{tabular}{l|l|l|l|l|l}
\hline Collection & Document set & \#docs & Size & \#query & \#qrels \\
\hline Robust04 & TREC Disks 4 \& 5 minus the Congressional Record & $528 \mathrm{k}$ & 1.9 GB & 249 & 17,412 \\
\hline WAPOST & TREC Washington Post Corpus & $608 \mathrm{k}$ & 6.9 GB & 50 & 3,948 \\
\hline
\end{tabular}

We explored different values of the window-size parameters in order to construct the term co-occurrence graph for each collection. Best results are obtained using a dynamic context window equal to sentence length.

All experiments were performed using the Terrier 4.2 IR platform ${ }^{3}$. We considered the title-description fields of the TREC topics as queries in our experiments. The preprocessing of both collections included stemming using the Porter stemmer and stopword removal using the default stopword list provided by Terrier. We applied the Okapi BM25 model with the default parameters used in Terrier to perform retrieval. The top 1000 documents are retrieved for each query.

Results are evaluated using four standard evaluation metrics, namely: mean average precision (MAP), normalized discounted cumulative gain (nDCG), precision at top 5 ranked documents (P@ 5) and precision at top 10 ranked documents (P@10). Additionally, statistical significance tests are performed using the two-tailed paired t-test at a 95\% confidence level (i.e., p_value < 0.05). Furthermore, we evaluate the robustness of methods using the standard robustness index (RI) (Collins-Thompson, 2009).

\subsection{Results and Discussion}

We now present experimental results to evaluate our proposal. We compare the effectiveness of our method against the baseline original queries, i.e. without reformulation. Besides, we consider three state-of-the-art PRF models, namely: Bo1 (Bose-Einstein 1), Bo2 (Bose-Einstein 2) and KLD (Kullback-Leibler Divergence) (Amati, 2003). For all PRF methods, we empirically set the number of expansion terms to 10. The results of our experiments on the two test collections are summarized in Tables 2 and 3. The superscripts 1/2/3/4 indicate that the improvements over the non-reformulated baseline, Bo1, Bo2 and KLD, respectively, are

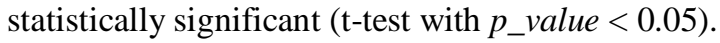

Table 2. Retrieval results on the TREC Robust04 collection

\begin{tabular}{l|c|c|c|c}
\hline Method & MAP & P@ 5 & P@ 10 & nDCG \\
\hline Baseline & 0.2515 & 0.4988 & 0.4325 & 0.5275 \\
\hline Bo1 & 0.2573 & 0.4996 & 0.4201 & 0.5373 \\
\hline Bo2 & 0.2378 & 0.4667 & 0.4048 & 0.5083 \\
\hline KLD & 0.2566 & 0.4892 & 0.4221 & 0.5350 \\
\hline BM25 $\operatorname{cog}$ & $0.2744^{1,2,3,4}$ & $0.5229^{1,3,4}$ & $0.4574^{1,2,3,4}$ & $0.5553^{1,2,3,4}$ \\
\hline
\end{tabular}

Table 3. Retrieval results on the TREC Washington Post collection

\begin{tabular}{l|c|c|c|c}
\hline Method & MAP & P@5 & P@ 10 & nDCG \\
\hline Baseline & 0.2346 & 0.4480 & 0.4440 & 0.5021 \\
\hline PRF_Bo1 & 0.2711 & 0.4480 & 0.4240 & 0.5356 \\
\hline PRF_Bo2 & 0.2766 & 0.4960 & 0.4500 & 0.5371 \\
\hline PRF_KLD & 0.2777 & 0.4720 & 0.4480 & 0.5426 \\
\hline BM25cog & $0.2638^{1}$ & $0.5120^{1}$ & 0.4740 & $0.5354^{1}$ \\
\hline
\end{tabular}

According to these tables, the proposed query reweighting method outperforms the non-reformulated baseline in all cases. The improvements in MAP and nDCG are always statistically significant, achieving $9.11 \%$ and $12.45 \%$ gains in terms of MAP in the Robust04 collection and the Washington Post collection,

\footnotetext{
${ }^{3}$ http://terrier.org/
} 
respectively. Similarly, we can see that the improvements in terms of precision are also statistically significant in both collections except in one case (P@10 in the Washington Post collection). These results indicate the effectiveness of the reweighting method based on BM25 $5_{\operatorname{cog}}$, which means that the important terms in the queries are assigned higher weights.

Analyzing the results obtained by the PRF methods, we observe in Table 4 that our method is outperformed in terms of MAP and nDCG in the TREC Washington Post collection. In this case, this shows that local analysis of pseudo-relevant documents is more effective than global analysis of the entire corpus. We believe that our results can be improved by using a local approach to construct the co-occurrence graph over the set of pseudo-relevant documents. Nevertheless, we can see that our method achieves better results on the standard TREC Robust 04 collection, with statistically significant improvements. The precision values obtained by the proposed method are higher than those achieved by the three PRF methods, in all cases, with statistical significant differences in the Robust04 collection. This shows that our proposal is more precise without adding new terms to the queries. Moreover, by comparing the PRF methods and the non-reformulated baseline, it can be observed that the precision is hurt on three out of four occasions in the Robust04 collection, which means that the added expansion terms in this case have a negative impact on precision.

To further investigate the effectiveness of our query reweighting method, we plot the interpolated precision-recall curves for the standard Robust04 collection in Figure 2. As it can be observed, the proposed query reweighting method clearly outperforms all other methods.

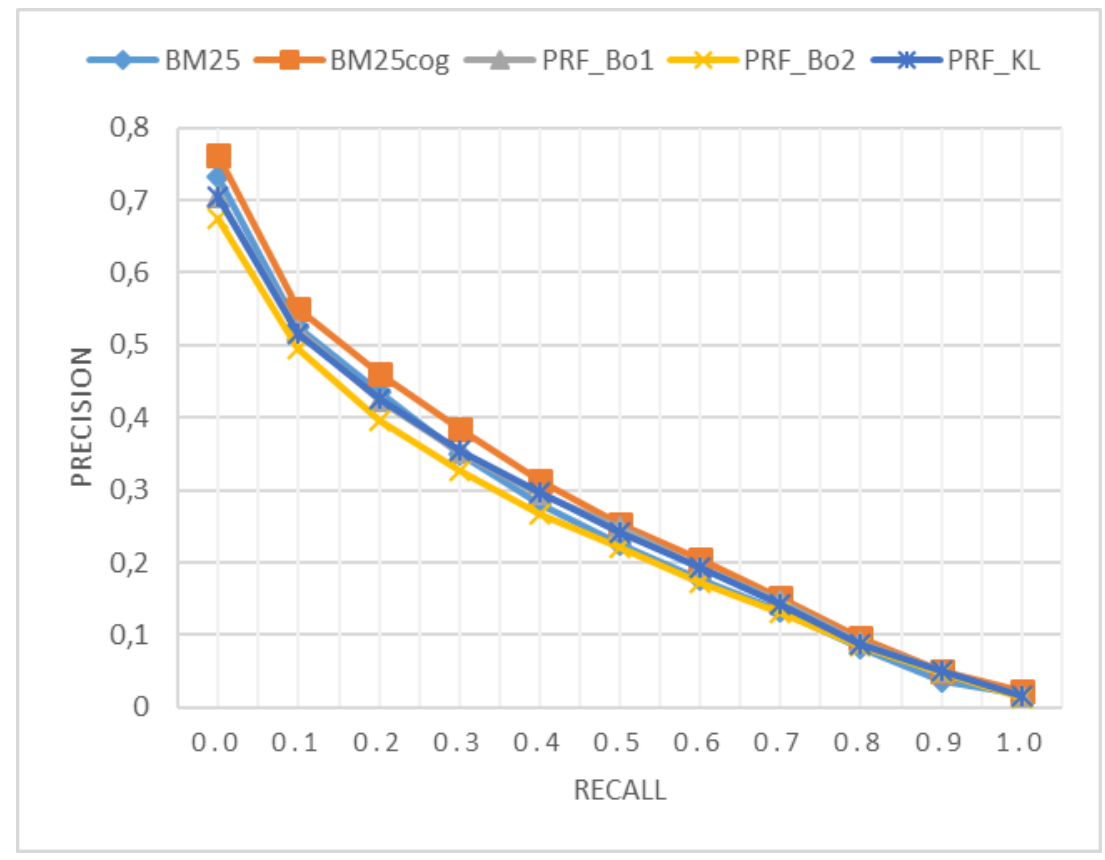

Figure 2. Interpolated precision-recall curves for the standard Robust04 collection

In terms of robustness, we report the RI (robustness index or reliability of improvement) for each method in Table 4. The RI measures the robustness of methods as follows (Collins-Thompson, 2009):

$$
R I(Q)=\frac{n_{+}-n_{-}}{|Q|} \in[-1,1]
$$

Where $Q$ is a set of queries, $\mathrm{n}_{+}$and $\mathrm{n}_{-}$are the number of improved and degraded queries, respectively, compared to the non-reformulated baseline and $|Q|$ is the total number of queries. A positive value of RI indicates that more queries were improved rather than degraded. According to the results in Table 4, we can see that our method obtains a largely higher robustness score than the PRF methods in the Robust04 collection and achieves similar values in the Washington Post collection. These results confirm that our proposal is shown to be both more effective and more robust than the state-of-the-art baseline methods. 
Table 4. Comparison of robustness index (RI) with respect to the non-reformulated baseline. Also shown are the actual number of improved queries by each method $\left(\mathrm{n}_{+}\right)$

\begin{tabular}{l|c|c|c|c|c|c|c|c}
\hline \multirow{2}{*}{ Collection } & \multicolumn{2}{|c|}{ Bo1 } & \multicolumn{2}{c|}{ Bo2 } & \multicolumn{2}{c|}{ KLD } & \multicolumn{2}{c}{ BM25 $\operatorname{cog}$} \\
\cline { 2 - 9 } & $\mathrm{n}_{+}$ & $\mathrm{RI}$ & $\mathrm{n}_{+}$ & $\mathrm{RI}$ & $\mathrm{n}_{+}$ & $\mathrm{RI}$ & $\mathrm{n}_{+}$ & $\mathrm{RI}$ \\
\hline Robust04 & 128 & +0.03 & 110 & -0.12 & 128 & +0.03 & 179 & +0.44 \\
\hline WAPOST & 32 & +0.28 & 28 & +0.12 & 32 & +0.28 & 32 & +0.28 \\
\hline
\end{tabular}

\section{CONCLUSION}

In this paper, we proposed an automatic query reweighting method based on term co-occurrence graphs. The latter are built over the entire document collection using a context window-based approach. The new weights of the query terms are calculated using an adapted version of the BM25 model, which allows to evaluate the discriminative power of terms and penalize trivial terms. Besides, it takes advantage of the BM25's adjusting parameters, which can be tuned to enhance retrieval results. Experiments on the standard TREC Robust04 collection and the new TREC Washington Post collection show that our proposal is both more effective and more robust than competitive state-of-the-art baselines.

As future work, we plan to investigate the use of a local approach for query reformulation, i.e., a co-occurrence graph built over pseudo-relevant documents. We believe that the results can be further improved, as similar studies have shown better results using a local approach. Another interesting research direction is to study the use of the model in a hybrid method for query expansion and reweighting.

\section{REFERENCES}

Aklouche, B., Bounhas, I., Slimani, Y., 2019a. Pseudo-Relevance Feedback Based on Locally-Built Co-occurrence Graphs. Proceedings of the 23rd European Conference on Advances in Databases and Information Systems. Bled, Slovenia, pp. 105-119.

Aklouche, B., Bounhas, I., Slimani, Y., 2019b. BM25 Beyond Query-Document Similarity. Proceedings of the 26th International Symposium on String Processing and Information Retrieval. Segovia, Spain.

Amati, G., 2003. Probability models for information retrieval based on divergence from randomness, Ph.D. Thesis, Department of Computing Science, University of Glasgow, United Kingdom.

Ariannezhad, M., Montazeralghaem, A., Zamani, H., Shakery, A., 2017. Improving Retrieval Performance for Verbose Queries via Axiomatic Analysis of Term Discrimination Heuristic. Proceedings of the 40th International ACM SIGIR Conference on Research and Development in Information Retrieval. Shinjuku, Tokyo, Japan, pp. 1201-1204.

Bendersky, M., Croft, W.B., 2008. Discovering Key Concepts in Verbose Queries. Proceedings of the 31st Annual International ACM SIGIR Conference on Research and Development in Information Retrieval. Singapore, Singapore, pp. 491-498.

Carpineto, C., Romano, G., 2012. A Survey of Automatic Query Expansion in Information Retrieval. ACM Computing Surveys (CSUR), 44(1), pp. 1-50.

Chang, Y.-C., Chen, S.-M., 2006. A new query reweighting method for document retrieval based on genetic algorithms. IEEE Transactions on Evolutionary Computation, 10(5), pp. 617-622.

Collins-Thompson, K., 2009. Reducing the Risk of Query Expansion via Robust Constrained Optimization. Proceedings of the 18th ACM Conference on Information and Knowledge Management. Hong Kong, China, pp. 837-846.

He, B., Huang, J.X., Zhou, X., 2011. Modeling term proximity for probabilistic information retrieval models. Information Sciences, 181(14), pp. 3017-3031.

Karisani, P., Rahgozar, M., Oroumchian, F., 2016. A query term re-weighting approach using document similarity. Information Processing \& Management, 52(3), pp. 478-489.

Kim, B.M., Kim, J.Y., Kim, J., 2001. Query term expansion and reweighting using term co-occurrence similarity and fuzzy inference. Proceedings Joint 9th IFSA World Congress and 20th NAFIPS International Conference (Cat. No. 01TH8569). Vancouver, BC, Canada, pp. 715-720.

Lv, Y., Zhai, C., 2011a. Lower-bounding Term Frequency Normalization. Proceedings of the 20th ACM International Conference on Information and Knowledge Management. Glasgow, United Kingdom, pp. 7-16. 
Lv, Y., Zhai, C., 2011b. When Documents Are Very Long, BM25 Fails!. Proceedings of the 34th International ACM SIGIR Conference on Research and Development in Information Retrieval. Beijing, China, pp. 1103-1104.

Mikolov, T., Sutskever, I., Chen, K., Corrado, G.S., Dean, J., 2013. Distributed Representations of Words and Phrases and their Compositionality. Proceedings of the 26th International Conference on Neural Information Processing Systems. Lake Tahoe, Nevada, United States, pp. 3111-3119.

Peat, H.J., Willett, P., 1991. The limitations of term co-occurrence data for query expansion in document retrieval systems. Journal of the American Society for Information Science, 42(5), pp. 378-383.

Rasolofo, Y., Savoy, J., 2003. Term Proximity Scoring for Keyword-based Retrieval Systems. Proceedings of the 25th European Conference on IR Research. Pisa, Italy, pp. 207-218.

Robertson, S., Zaragoza, H., 2009. The Probabilistic Relevance Framework: BM25 and Beyond. Foundations and Trends in Information Retrieval, 3(4), pp. 333-389.

Robertson, S.E., Jones, K.S., 1976. Relevance weighting of search terms. Journal of the American Society for Information Science, 27(3), pp. 129-146.

Robertson, S.E., Walker, S., 1994. Some Simple Effective Approximations to the 2-Poisson Model for Probabilistic Weighted Retrieval. Proceedings of the 17th Annual International ACM SIGIR Conference on Research and Development in Information Retrieval. Dublin, Ireland, pp. 232-241.

Salton, G., Buckley, C., 1988. Term-weighting approaches in automatic text retrieval. Information Processing \& Management, 24(5), pp. 513-523.

Song, R., Taylor, M.J., Wen, J.-R., Hon, H.-W., Yu, Y., 2008. Viewing Term Proximity from a Different Perspective. Proceedings of the IR Research. Proceedings of the 30th European Conference on Advances in Information Retrieval. Glasgow, United Kingdom, pp. 346-357. 